Article

\title{
Two-Dimensional Imaging of Permittivity Distribution by an Activated Meta-Structure with a Functional Scanning Defect
}

\author{
Go Itami ${ }^{1,2, *(1)}$, Osamu Sakai ${ }^{1,2}$ and Yoshinori Harada ${ }^{3}$ \\ 1 Department of Electronic Systems Engineering, The University of Shiga Prefecture, Shiga 522-8533, Japan; \\ sakai.o@e.usp.ac.jp \\ 2 Electric Science and Engineering, Kyoto University, Kyoto 615-8510, Japan \\ 3 Graduate School of Medicine, Kyoto Prefectural University of Medicine, Kyoto 602-8566, Japan; \\ yoharada@koto.kpu-m.ac.jp \\ * Correspondence: ot68gitami@ec.usp.ac.jp
}

Received: 31 January 2019; Accepted: 18 February 2019; Published: 20 February 2019

\begin{abstract}
A novel 2D imaging method for permittivity imaging using a meta-structure with a functional scanning defect is proposed, working in the millimeter wave-range. The meta-structure we used here is composed of a perforated metal plate with subwavelength-holes and a needle-like conductor that can scan two-dimensionally just beneath the plate. The metal plate, which is referred to as a metal hole array (MHA) in this study, is known as a structure supporting propagation of spoof surface plasmon polaritons (SSPPs). High-frequency waves with frequencies higher than microwaves, including SSPPs, have the potential to detect signals from inner parts embedded beneath solid surfaces such as living cells or organs under the skin, without physical invasion, because of the larger skin depth penetration of millimeter wave-bands than optical wave-bands. Focused on activated SSPPs, the localized distortion of SSPP modes on an MHA is used in the proposed method to scan the electromagnetic properties of the MHA with a needle-like conductor (conductive probe), which is a kind of active defect-initiator. To show the validity of the proposed method, electromagnetic analyses of the localized distortions of wave fields were performed, and one- and two-dimensional imaging experiments were conducted with the aim of detecting both conductive and dielectric samples. The analytical results confirmed the localized distortion of the electric field distribution of SSPP modes and also indicated that the proposed method has scanning ability. In experimental studies, the detection of conductive and dielectric samples was successful, where the detected dielectrics contained pseudo-biological materials, with an accuracy on the order of millimeters. Finally, a biomedical diagnosis in the case of a rat lung is demonstrated by using the experimental system. These results indicate that the proposed method may be usable for non-invasive and low-risk biomedical diagnosis.
\end{abstract}

Keywords: spoof surface plasmon polariton (SSPP); metal hole arrays (MHA); electromagnetic distortion; two-dimensional imaging

\section{Introduction}

Electromagnetic waves have huge potential for various applications such as wireless communication, micro processing, and sensing, and as a result, they will become indispensable in our daily lives. In recent years, millimeter waves or terahertz waves, with wavelengths shorter than microwaves and longer than infrared, have been attracting considerable attention. One of the features of these waves is their combination of high resolution and non-destructiveness. Therefore, a number of imaging methods that use these waves have been reported [1-3]. However, since these waves generally propagate in 
straight lines and attenuate immediately in lossy media, such methods frequently encounter technical issues due to the deterioration of information-carrying detection signals and their physical separation due to multipath propagation. From a microscopic point of view, an electromagnetic wave includes two components: electric and magnetic fields with their spatial distributions varying in time. This is significantly different from other waves, like sonic waves, which can be interpreted in terms of single-variable wave propagation.

When we impose specific boundary conditions on electromagnetic waves, there is a wave component with a wavenumber vector that does not propagate spatially and concentrates around the boundaries or interfaces with exponential attenuation. This phenomenon corresponds to an evanescent wave, and in classical cases it has only been observed in the optical region [4]. In a microscopic view, when the evanescent waves get close to a conductive plate, oscillations are excited in the free electrons in the plate surface. This phenomenon is called a surface plasmon polariton (SPP) or simply, a surface wave [5-8], and it has been used in applications such as biosensors, chemical sensors and field enhancement in spectroscopy [9-11]. As described above, although an SPP can be excited only in the optical region, a similar phenomenon, with the concentration and oscillation of electric fields on the surface of a conductive plate, has also been confirmed at lower frequencies than optical bands. These are called spoof surface plasmon polaritons (SSPP) [12-16], and are broadly considered to be a unique phenomenon involving a metamaterial. One of the features of SSPPs that is not observed in other propagating modes, is the concentration of its electric field at interfaces, so that it has the potential to give us significant information about objects near such surfaces without complex signal processing. The standard example of an SSPP structure is a metal hole array (MHA), which is a conductive plate with periodic holes [17-20]. An MHA is considered to be a macroscopic effective medium in terms of controlling relative permittivity by the structural parameters of its holes. Since its structure has only a few parameters, an MHA is expected to serve as a ready-to-use functional and practical medium as well as a readily available sample for experiments [21]. Therefore, many studies of SSPPs on MHAs have been reported, although so far, they have focused on static properties as SSPP applications.

In this study, a two-dimensional imaging method using an MHA as a dynamic functional material is proposed for permittivity imaging, mainly aimed at a non-invasive medical diagnostic. Specifically, the proposed method uses the intentional formation of electromagnetic defects in the uniform electric distributions of SSPPs on an MHA, where the defect is formed by scanning a metallic needle across the surface of the MHA. Viewed another way, the proposed method is also a non-destructive diagnostic method used as a dynamic dielectric sensor for an MHA, which is sensitive to the side opposite the needle-scanned surface. Compared to other non-destructive diagnosis tools such as confocal microscopy and near-field optical microscopy, an advantage of the proposed method is that the electromagnetic properties of subsurface tissues can be measured because surface waves in millimeter or terahertz wave bands have deeper skin penetration depths than those in optical frequency bands [22-24]. At present, the imaging of inner issues is typically achieved using a destructive method such as CT (Computer Tomography) or some other high-cost technique such as MRI (Magnetic Resonance Imaging). Actually, conventional imaging methods face difficulties arising from these issues [25-27]. Biomedical imaging based on the electromagnetic properties of biomedical tissues is considered to be an effective method because the electromagnetic properties of a malignant tumor and normal breast tissue are different [28].

In Section 2, the excitation mechanism of SSPPs on an MHA is described. In Section 3, the numerical analysis of the electromagnetic properties of an MHA, such as transmittance and reflectance, and the localized distortion of the electric fields of the SSPP mode around the MHA with a conductive probe (a needle-like conductor) is demonstrated. In Section 4, we report one- and two-dimensional imaging experiments including the experimental demonstration of biomedical diagnosis in the case of a rat lung that apply the localized distortion mechanism, with the use of an MHA targeting conductive, dielectric, and biomedical samples. 


\section{The Basis of Spoof Surface Plasmon Generation}

The SSPP phenomenon was discovered by Pendry et al. who also derived the theory of its generation. Here, SSPP is used for imaging by detecting the local deformation of its propagation modes. Therefore, in order to explain the proposed imaging method, we begin with the theoretical derivation of SSPP generation as its basis. First of all, the mechanism of SSPP generation on an MHA is explained. In order to describe SSPP generation on an MHA, the electromagnetic model of the process is introduced. In this study, the MHA has holes whose size is the same order of magnitude as the wavelengths of incident waves. The model is shown in Figure 1. In this model, the MHA is treated as a uniform, effective medium with a frequency-based response to incident waves. As shown in Figure 1, we consider that with the incidence of optimized millimeter waves, electric fields concentrate in and around an MHA that has two-dimensional waveguide arrays (period $d$, cross section $p \times q$, depth $w$,) extended to infinity. It is assumed that the incident waves are TM-polarized optimal waves. Note that the electric field-vector of the wave has only $x$ - and $z$-components, and the magnetic field-vector has only $y$-component in the situation. In the derivations, the coupling phenomenon between the dispersion relation of the incident waves and the waveguide modes is considered. That is, the electric fields are considered to be concentrated on the surface, and this distribution forms longitudinal waves. Thus, the SSPP modes are generated on the MHA.

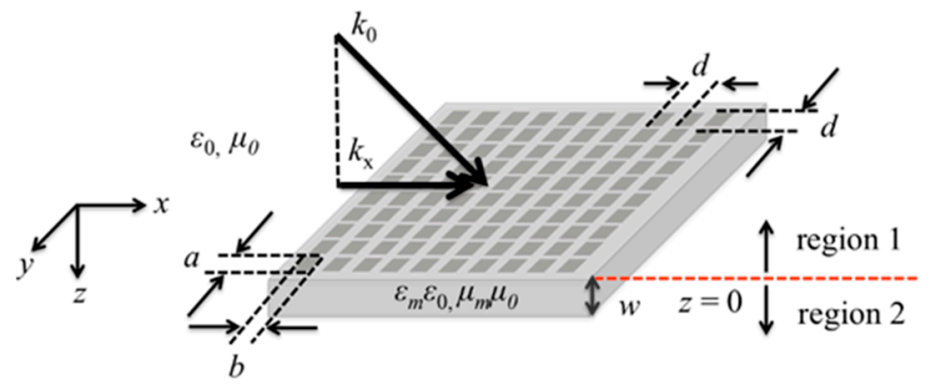

Figure 1. The theoretical model of the metal hole array (MHA) with TM incident waves. Region 1 is the space above the MHA. Region 2 is the space in the holes of the MHA.

Before beginning the derivation of SSPP generation, the definitions of the electric and magnetic properties in two regions are introduced. In Figure 1, Region 1 indicates the space on the upper side of the MHA, and Region 2 indicates the space inside the holes of the MHA. Since Region 1 is filled with vacuum, the permittivity and the permeability in Region 1 are $\varepsilon_{0}$ and $\mu_{0}$ respectively. On the other hand, as described above, since the MHA is presumed to be a macroscopically uniform medium, the MHA has to have an effective relative permittivity of $\varepsilon_{m}$ and a relative permeability $\mu_{m}$ that is frequency dependent [12]. When electromagnetic waves whose frequency is below a cut-off frequency are introduced to an MHA, waves are attenuated with fundamental mode [29]. Therefore, the electric fields of incident waves are expressed as below.

$$
E_{x}=E_{0} \sin \left(\frac{\pi y}{a}\right)
$$

Note that in this manuscript, time oscillation terms are omitted for convenience of formulation if necessary. Here, $E_{0}$ is a constant value, and expression (1) indicates that $E_{x}=0$ at the edges of the holes. Therefore, the $x$-component and $z$-component of an incident wavevector $k_{x}$ and $k_{z}$ can be expressed as

$$
\begin{gathered}
k_{x}=\frac{\pi}{a} \\
k_{z}=\sqrt{\left(\frac{\pi}{a}\right)^{2}-\omega^{2} \varepsilon_{\mathrm{h}} \mu_{\mathrm{h}} \varepsilon_{0} \mu_{0}}
\end{gathered}
$$


where $\varepsilon_{\mathrm{h}}$ and $\mu_{\mathrm{h}}$ are respectively, the relative permittivity and relative permeability in the holes of the MHA. At this point, remembering the precondition that the MHA is a macroscopic medium and has an effective relative permittivity, for example, when focused on a unit cell of the MHA (shown in Figure 2), the macroscopic wave vector only has a component in the $z$-direction $k_{z}{ }^{\prime}$ because the waveguide mode occurs only in the holes and provides no propagation in the $x$ - and $y$-directions. From the above discussions, $k_{z}{ }^{\prime}$ can be expressed as below.

$$
k z_{z}=k_{0} \sqrt{\varepsilon_{x} \mu_{y}}=k_{0} \sqrt{\varepsilon_{\mathrm{m}} \mu_{\mathrm{m}}}, k_{0}=\frac{\omega}{c_{0}}
$$

where $c_{0}$ is the velocity of light in a vacuum. The wave number of $k_{z}{ }^{\prime}$ and that of $k_{z}$ should be essentially the same value, because the unit cell in Figure 2 has one waveguide and there is no propagation in the conductive medium of the MHA. Therefore, the equation below is obtained by using the expression $k_{z}=k_{z}{ }^{\prime}$.

$$
\frac{\omega}{c_{0}} \sqrt{\varepsilon_{\mathrm{m}} \mu_{\mathrm{m}}}=\sqrt{\left(\frac{\pi}{a}\right)^{2}-\omega^{2} \varepsilon_{\mathrm{h}} \mu_{\mathrm{h}} \varepsilon_{0} \mu_{0}}
$$

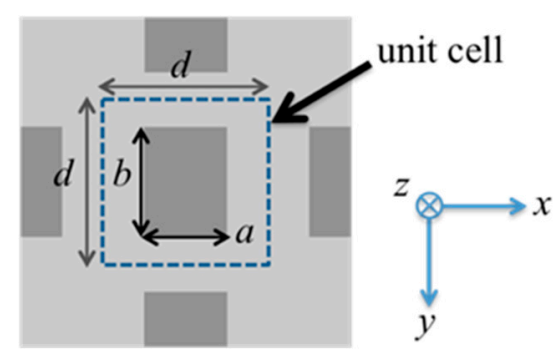

Figure 2. The unit cell of the MHA.

On the other hand, in the matching procedure at $z=0$, the instantaneous flow of energy across the surface has to be considered by treating the unit cell as both a microscopic and a macroscopic medium. With the average electric fields of the unit cell regarded as a macroscopic medium

$$
\begin{aligned}
\overline{E_{0}}= & E_{0} \frac{a}{d^{2}} \int_{0}^{b} \sin \left(\frac{\pi y}{a}\right) d y=\frac{2 a b}{\pi d^{2}} E_{0} \\
(\boldsymbol{E} \times \boldsymbol{H})_{\mathrm{z}, \mathrm{mic}}= & \frac{-k_{z} E_{0}^{2}}{\omega \mu_{\mathrm{h}} \mu_{0}} \frac{a}{d^{2}} \int_{0}^{b} \sin ^{2}\left(\frac{\pi y}{a}\right) d y=\frac{-k_{z}}{\omega \mu_{\mathrm{h}} \mu_{0}} E_{0}{ }^{2} \frac{a b}{2 d^{2}} \\
& (\boldsymbol{E} \times \boldsymbol{H})_{\mathrm{z}, \mathrm{mac}}=\frac{-k_{z}{\overline{E_{0}}}^{2}}{\omega \mu_{\mathrm{m}} \mu_{0}}
\end{aligned}
$$

Since the two expressions (7) and (8) for the instantaneous flow of energy across the surface must have the same value, the equation $(\boldsymbol{E} \times \boldsymbol{H})_{\mathrm{z} \text {, mic }}=(\boldsymbol{E} \times \boldsymbol{H})_{\mathrm{z} \text {, mac }}$ is always useful. With this equation and expressions (5)-(8), the effective relative permittivity $\varepsilon_{\mathrm{m}}$ and relative permeability $\mu_{\mathrm{m}}$ can be obtained as below.

$$
\begin{gathered}
\mu_{\mathrm{m}}=\mu_{\mathrm{h}} \frac{8 a b}{\pi^{2} d^{2}} \\
\varepsilon_{\mathrm{m}}=\frac{\pi^{2} d^{2}}{8 a b}\left[1-\left(\frac{\omega_{\mathrm{p}}}{\omega}\right)^{2}\right], \omega_{\mathrm{p}}=\frac{\pi c}{\sqrt{\varepsilon_{\mathrm{h}} \mu_{\mathrm{h}}} a}
\end{gathered}
$$

where $\omega_{\mathrm{p}}$ is a cutoff frequency, and $\varepsilon_{\mathrm{h}}$ is the relative permittivity and $\mu_{\mathrm{h}}$ is the relative permeability in the holes of the MHA. Expression (10) confirms that the effective relative permittivity of the MHA is frequency dependent. Thus, the MHA can be treated as an electromagnetic functional material.

On the other hand, remembering the magnetic field of the incident waves, this can be also solved by a wave equation for the value of $H_{y}$. Here, in discussing the generation of SSPPs in the MHA, 
we start by considering the magnetic field near the MHA. First, the wave equation for $H_{y}$ is obtained by considering the Maxwell equations.

$$
\left(\frac{\partial^{2}}{\partial x^{2}}+\frac{\partial^{2}}{\partial z^{2}}\right) H_{y}=-\omega^{2} \varepsilon_{\mathrm{r}} \mu_{\mathrm{r}} \varepsilon_{0} \mu_{0} H_{y}
$$

where $\varepsilon_{\mathrm{r}}$ and $\mu_{\mathrm{r}}$ are the relative permittivity and the relative permeability. With the precondition that the TM wave is incident to the MHA, it is considered that if SSPPs occur on the surface of the MHA, they must propagate along the $x$-direction. Also, since the incident magnetic field has the propagation constants of $x$ and $z$, the y component of the magnetic field vector $H_{y}$ can be expressed thus,

$$
H_{y}=h(z) \exp \left[j\left(k_{\|} x-\omega t\right)\right]
$$

where $k_{\mid}$। is the wavenumber of an SSPP, and $h(z)$ is the amplitude of the magnetic field, depending only on the value of $z$. Substituting expression (12) into expression (11), the equation for $h(z)$ is obtained. It has the form

$$
\frac{\partial^{2}}{\partial z^{2}} h(z)=\left(k_{i}^{2}-\omega^{2} \varepsilon_{\mathrm{r}} \mu_{\mathrm{r}} \varepsilon_{0} \mu_{0}\right) h(z)
$$

where $k_{i}$ is the wavenumber of the magnetic field. In Region $1, k_{1}=\left(\left.k_{\mid}\right|^{2}-\omega^{2} \varepsilon_{0} \mu_{0}\right)^{1 / 2}$, in Region 2, $k_{2}=\left(-\omega^{2} \varepsilon_{\mathrm{m}} \mu_{\mathrm{m}} \varepsilon_{0} \mu_{0}\right)^{1 / 2}$. In Region 2, when regarding the MHA as a macroscopic medium, there is no propagation of the EM waves in $x-y$ directions, and $k_{1}$ and $k_{2}$ are required to have positive values. Considering the solution of equation (13), as the value of $|z|$ increases, the value of the amplitude $h(z)$ is supposed to decrease exponentially. From these assumptions, the relative equation obtained is:

$$
\text { At } z<0
$$

$$
h(z)=h_{1} \exp \left(k_{1} z\right)
$$

On the other hand, at $z \geq 0$

$$
h(z)=h_{2} \exp \left(-k_{2} z\right)
$$

When considering the boundary condition of the electromagnetic field and magnetic field between Region 1 and Region 2 (at $z=0$ ), the tangent components of the electric field and the magnetic field in Region 1 and Region 2 must have the same value. This condition corresponds to the condition of conventional surface plasmon generation, that is

$$
k_{1}=-\frac{k_{2}}{\varepsilon_{\mathrm{m}}},\left(h_{1}=h_{2}\right)
$$

The above expression is often used to derive the dispersion relation of a surface plasmon. However, expression (16) can also be used to derive the dispersion relation of the SSPP. By squaring expression (16) and using expression (10) and the values of $k_{1}$ and $k_{2}$, the dispersion relation that is fundamental to comprehending the results in this paper is obtained. That is,

$$
k_{\|}^{2} c_{0}^{2}=\omega^{2}+\frac{\omega^{4}}{\omega_{\mathrm{p}}^{2}-\omega^{2}}\left(\frac{8 a b}{\pi^{2} d^{2}}\right)^{2}
$$

Note that the formula $c^{2}{ }_{0}=1 / \omega^{2} \varepsilon_{0} \mu_{0}$ is used when deriving expression (17). The above expression is called the SSPP dispersion relation. The curve of the dispersion relation is shown in Figure 3. If an angular frequency $\omega$ is close to the cutoff frequency $\omega_{\mathrm{p}}$, the wavenumber $k_{\mid}$। diverges to infinity. In other words, the SSPP modes stop propagating then. On the other hand, as the value of $\omega$ approaches zero, the dispersion relation curve comes close to a light line, although they do not cross each other, as shown in Figure 3. Therefore, if an actual dispersion relation corresponds to this curve, SSPP modes are not generated. However, since the MHA is a periodic structure, it has to be treated as a two-dimensional crystal. Therefore, it should be considered that lattice scattering effects are occurring 
in the MHA. Here, introducing the reciprocal lattice of its structure, the definition of the wavenumber $k_{1}$, can be replaced as

$$
\boldsymbol{k}_{\|}^{\prime}=\boldsymbol{k}_{\|}-\mathbf{i} \boldsymbol{G}_{x}-\mathbf{j} \boldsymbol{G}_{y}, \quad\left|\boldsymbol{G}_{x}\right|=\left|\boldsymbol{G}_{y}\right|=\pi / d
$$

where $G_{x}$ and $G_{y}$ are the reciprocal lattice vectors in the $x$-direction and $y$-direction, respectively. So, the dispersion relation of the MHA in infinite space is also replaced by one with the spatial periodicity shown in Figure 4. Figure 4 confirms that there are intersection points between the actual dispersion relation and a light line. Therefore, SSPPs are indeed generated in the MHA, especially when the angular frequency $\omega$ is close to the cutoff frequency $\omega_{\mathrm{p}}$. If the SSPPs are generated on the surface of the incidence side of the MHA, the waves are also generated on the opposite side because the waves transmitted through the waveguides of the MHA also couple with the SSPP mode in the same way as with the incident waves. The important feature of an SSPP mode is the concentration of the electric field on the MHA. Therefore, we focused on a modification of the concentrated electric fields for a novel use as an imaging method.

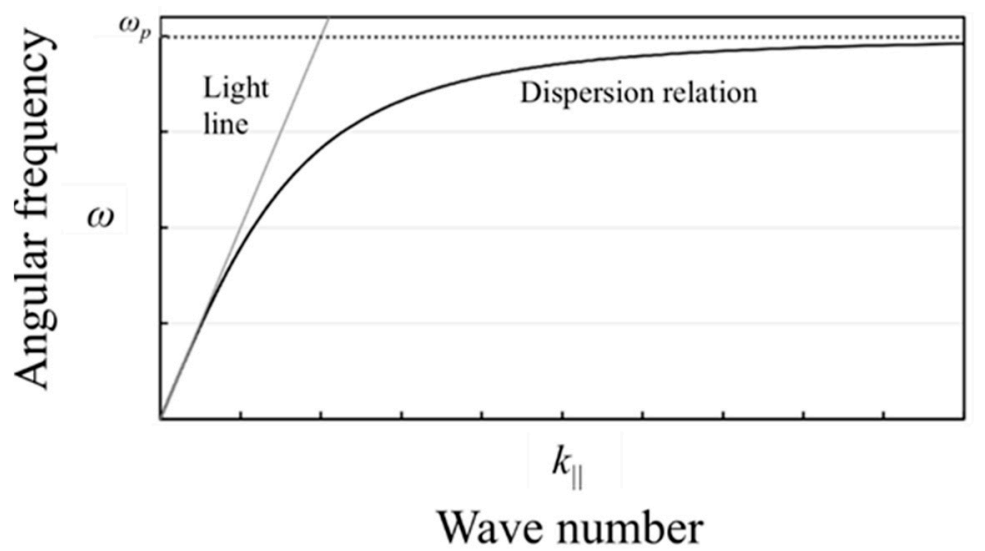

Figure 3. The dispersion relation of spoof surface plasmon polaritons (SSPP).

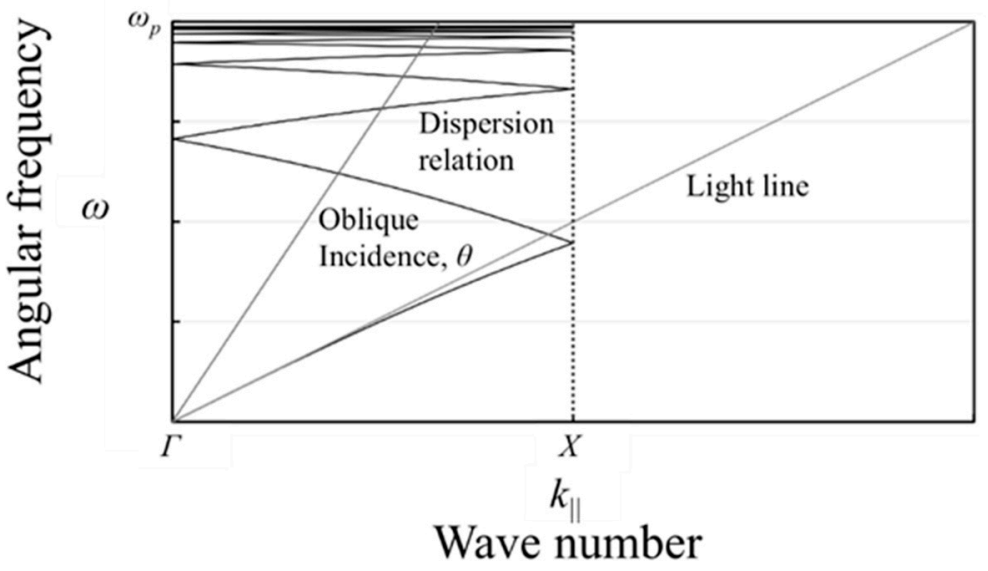

Figure 4. The real dispersion relation of SSPP with lattice scattering effects.

\section{Electromagnetic Numerical Analyses of The MHA and Electromagnetic Distortion}

In Section 2, we overviewed the physical basis of SSPP propagation and its dispersion, which showed that its frequencies are in fact caused and assured by the MHA. The concentration of its fields around the propagation interface was also pointed out, leading to potential applications of this propagation mode to $2 \mathrm{D}$ permittivity imaging that requires field uniformity over the area to be surveyed. However, the SSPP itself cannot provide us with local information on its responses to an electric field or permittivity, so in this section we confirm the effects of defect introduction on SSPP propagation. 
Electromagnetic numerical analyses were performed to reconfirm the electromagnetic properties of the MHA predicted in Section 2 and to specify the electromagnetic field distortion on the MHA by a needle-like conductor. Figure 5 shows the analytical model using an electromagnetic simulator (HFSS R18, Ansys, Canonsburg, PA, USA). In this simulation model, the MHA is made of copper and its thickness is $2 \mathrm{~mm}$. The size of the embedded waveguide is $2 \mathrm{~mm} \times 1.5 \mathrm{~mm}$ and the period in both the $x$ - and $y$-directions is $3 \mathrm{~mm}$. The coordinate notations used here correspond to those in Section 2 . Periodic boundary conditions applied to the sides of the model yield a hypothetical infinite area of SSPP propagation, and the incident surface (underside, port 1) is on the side opposite the receiving surface (topside, port 2) along the long side of the model, which explains the macroscopic wave propagation; they are defined as a Floquet port. Assuming the dispersion relation shown in Figure 4 for the MHA, SSPP modes exhibit resonant frequencies in a range lower than the cutoff frequency. To confirm this prediction, $S$-parameters $\left(S_{11}\right.$ and $\left.S_{21}\right)$ are analyzed under the above conditions in our numerical calculation, as shown in Figures 6 and 7.

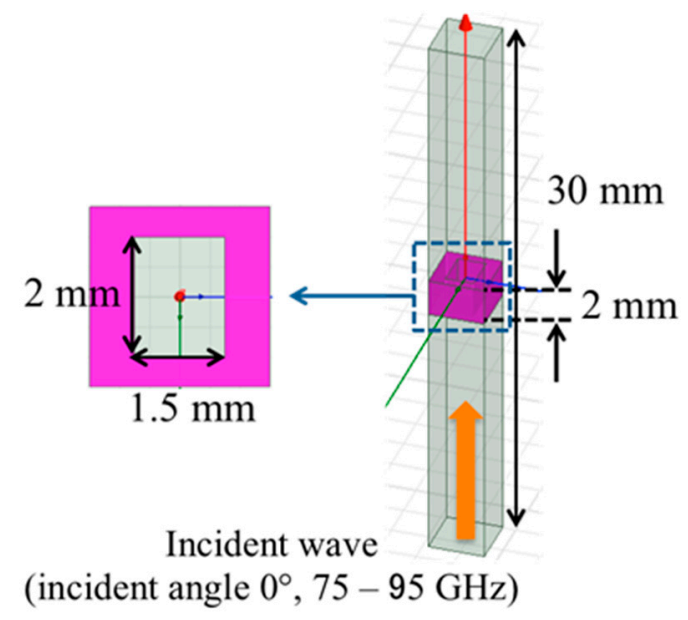

Figure 5. The MHA model for numerical analysis of the frequency characteristics of reflectance and transmittance.

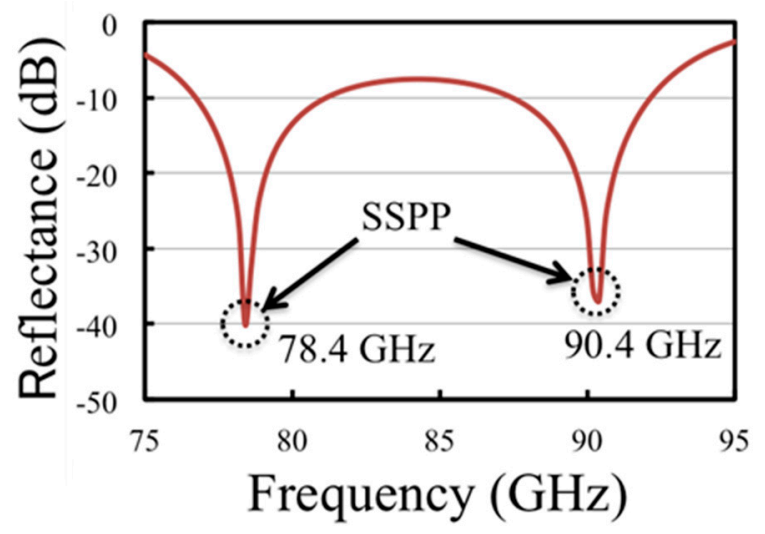

Figure 6. The frequency dependence of the reflectance of the MHA.

In Figures 6 and 7, the frequency spectrum of the transmission rate has peaks around $78.4 \mathrm{GHz}$ and $90.4 \mathrm{GHz}$. This indicates the existence of at least two resonant frequencies in the MHA. These two resonances coincide with the intersection points of the dispersion relation of the SSPP and the light line in Figure 4; at these frequencies, energy conversion from the mode in free space propagation to the SSPP mode is smooth, thanks to good wave matching conditions. This phenomenon was termed extraordinary transmission as one of the features of an MHA [17-21]. In other words, at $78.4 \mathrm{GHz}$ and $90.4 \mathrm{GHz}$, SSPP generation/propagation is possible where SSPP modes are able to uniformly 
concentrate electric fields on and in the vicinity of MHAs due to the multiple lattice scattering described in Equation (18).

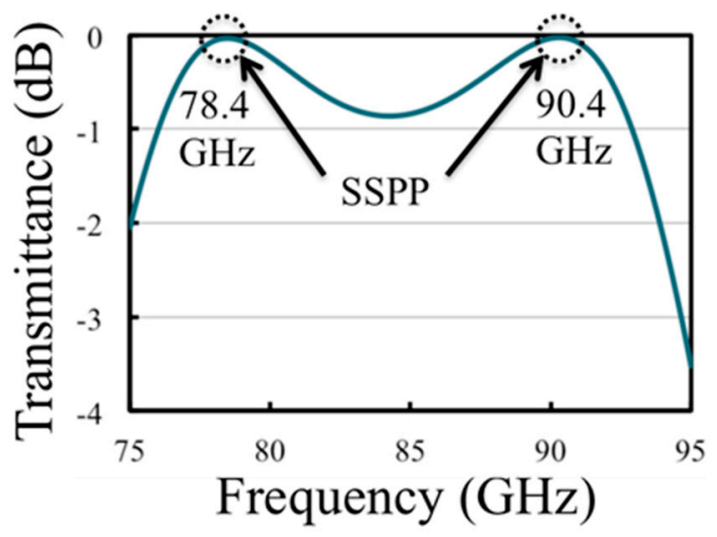

Figure 7. The frequency dependence of the transmittance of the MHA.

In this uniform 2D field, localized distortion is induced by inserting a needle-like conductor into a hole of the MHA. Electric fields are distorted strongly at sharp conductive boundaries, a phenomenon known as the near field effect [30], unlike wave propagation that includes a dispersion relation. Such distorted electric fields decay spatially with a wavelength-order attenuation constant. For this reason, another set of two analytical models (shown in Figure 8) was tested in our simulation runs. One has the MHA equipped with a conductive probe, and the other has only the MHA with its periodic 2D structure. That is, although the shape of both MHAs is the same as that in the model in Figure 5, the model has $5 \times 5$ holes in order to observe the difference in electric field distributions between case 1 , insertion of a conductive probe in the MHA, and case 2, removal of the conductive probe. Note that the model of a conductive probe is positioned in or near a center hole. The probe has a cone shape, with a radius at the bottom plane of $0.5 \mathrm{~mm}$ and a height of $6 \mathrm{~mm}$. Under these conditions, by using the model as shown in Figure 8, the electric field distributions of the two cases were monitored at $78.4 \mathrm{GHz}$, which is a candidate for the SSPP frequency near the resonant frequency observed in Figure 6. The results of the distributions in the two cases and their numerical comparison at $\mathrm{z}=+0.5 \mathrm{~mm}$ and $-0.5 \mathrm{~mm}$, are shown in Figure 9 .
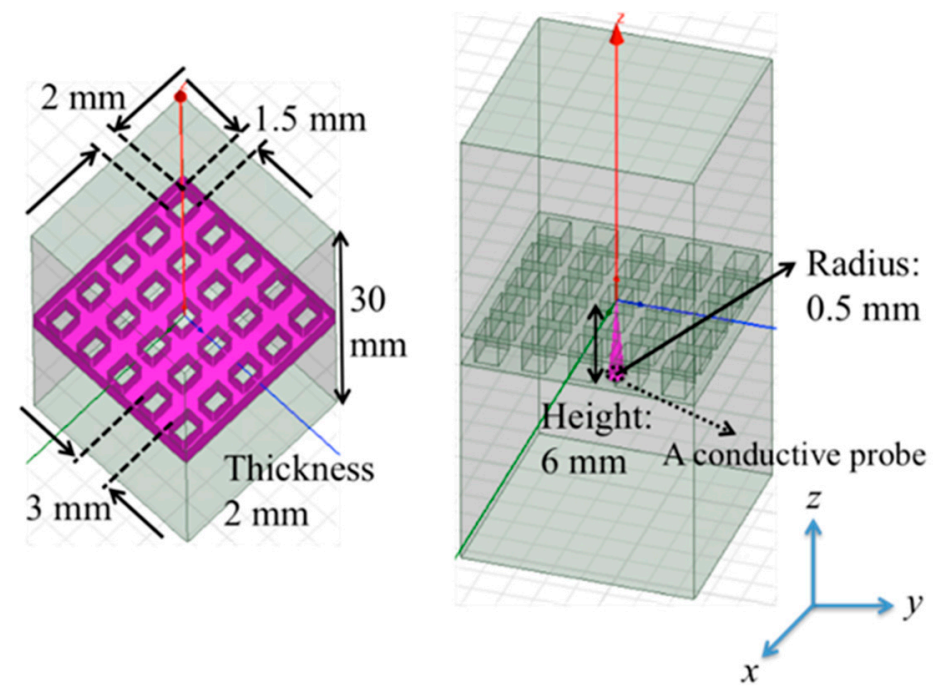

Figure 8. The two models for numerical analyses of the electric field distribution around the MHA. 


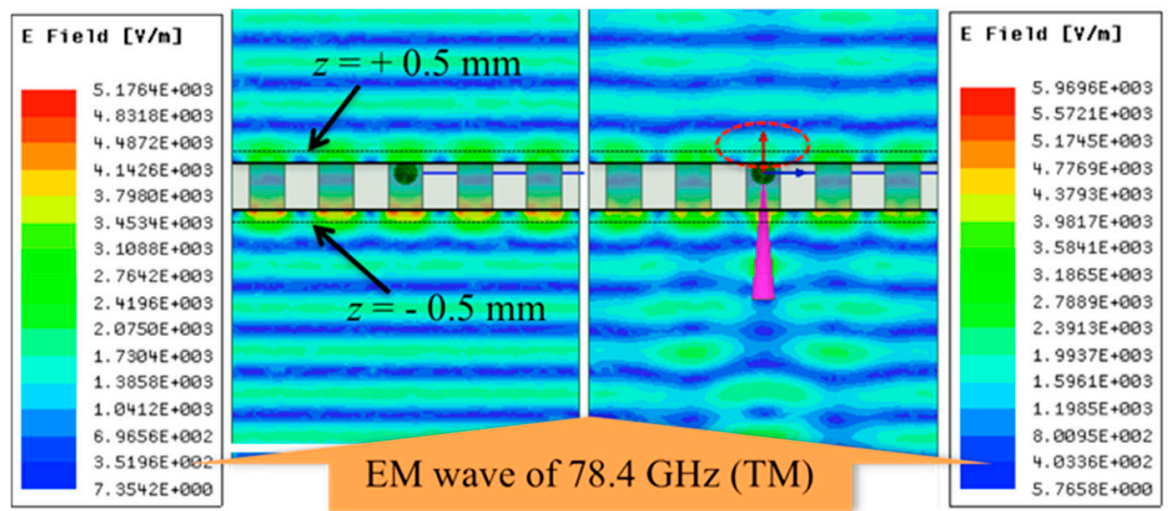

(a)

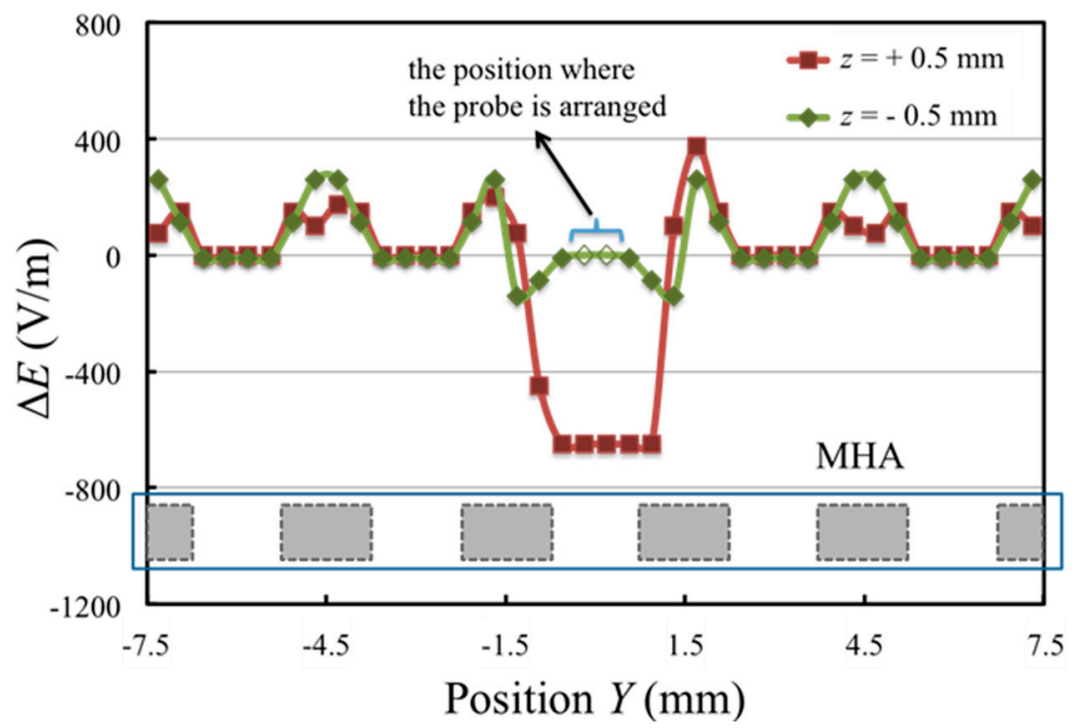

(b)

Figure 9. (a) The two electric field distributions around the MHA without (left) and with (right) a conductive probe; (b) the numerical results of the two cases $(z=+0.5,-0.5 \mathrm{~mm})$, which is the difference between the distributions with and without a conductive probe.

From Figure 9a, in the case on the left, electric fields are concentrated around the MHA, which is unlike the electric fields of the waveguide mode because those fields are concentrated not around holes but in holes. However, the results indicate that the fields are like compression waves, strongly affected by existing holes. Since the distribution of the fields around the MHA is symmetrical in the $y-z$ plane, the electric-field distributions are partial components of an SSPP. This fact is consistent with a feature of SSPPs in which the mode expands its field on the side opposite the incident side when the mode is excited at an incident side. This result shows that the fields distribute by a coupling phenomenon between the SSPP mode and transmission waves. On the other hand, in the case on the right, looking at the electric distribution in the red circle, it was found that the electric field distributions are less concentrated than the distributions at the same region in the case on the left, while field distributions of the other holes in the case on the right are at the same level as those in the case on the left. These facts indicate that the electric fields in the region with the red circle are locally distorted by a conductive probe. Consequently, the results specifically show that localized electromagnetic distortions can be generated by inserting a conductive probe in a hole of an MHA. 
From Figure 9b, as shown in the numerical results in the case of $z=+0.5 \mathrm{~mm}$ in Figure $9 \mathrm{a}$, it was found that there are significant differences in the region around a conductive probe. This is because the electric field distribution on the MHA is deformed by the insertion of a conductive probe, as displayed in Figure 9a. On the other hand, from the numerical results in the case of $z=-0.5 \mathrm{~mm}$, it was found that the difference between the two electric field distributions under the MHA is much smaller than the one on the MHA. This might be explained by the suggestion that the field distributions around the MHA are only distorted by a near-field effect. From these results, the differences in the two cases are potentially sufficient to detect the information in the deformed distributions because in practice, these differences are detected as signal intensities related to the square of the electric field.

The discussion above suggests that by taking the difference between the electric-field distributions of the holes when a conductive probe is inserted, and when not, local signals in the limited vicinity of each hole of the MHA can be obtained with detectable intensities and enhanced spatial resolution.

\section{Two-Dimensional Imaging Experiments Using MHA and a Conductive Probe}

In Section 3, the numerical results showed that an SSPP propagates in the MHA when electromagnetic waves at optimal frequencies enter the plate, and that its propagation can be locally deformed by a conductive metallic probe. To detect wave media information at the deformation point where electromagnetic waves enter the MHA, scattered waves generated apart from reflected waves play important roles in the frequency range, which includes the incidence of optimal waves. In the experiments discussed in this section, we used scattered waves, which exhibit the characteristics of local electromagnetic distribution [31]. Transmitter and receiver antennae, and a conductive probe are the main diagnostic tools manipulated for signal detection.

We performed experiments on one- and two-dimensional imaging using an MHA with structural parameters that were the same as those in the analytical model, and the MHA was equipped with a scanning conductive probe. After the one-dimensional imaging experiments, two-dimensional imaging experiments were performed using conductive and dielectric samples. Finally, we demonstrated a biomedical diagnosis in the case of a rat lung by using the system. The experimental system is shown in Figures 10-12, with a schematic view of the corresponding MHA and the conductive probe.

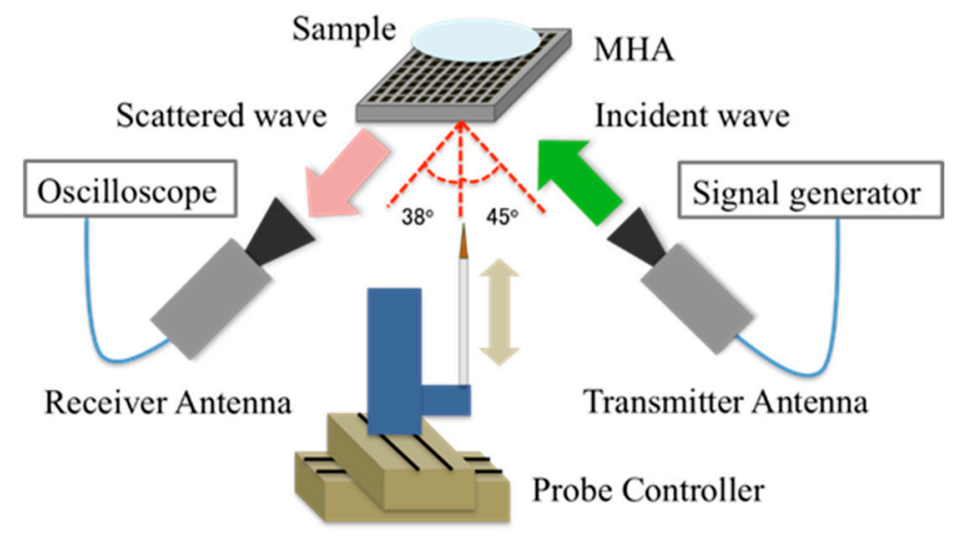

Figure 10. The experimental system for one- and two- dimensional imaging experiments.

As suggested by the above analyses, we searched for the most appropriate frequency for electromagnetic-wave experiments in the optimal bands for SSPP propagation, by using one-dimensional imaging experiments, which gave us simple and straightforward evidence as the conductive probe scanned along one line. With the incidence of electromagnetic waves at $78-86 \mathrm{GHz}$ from a transmitter antenna into the structure shown in Figure 11, SSPPs form on the MHA and scattered waves are emitted, partly at the reflection angle of 38 degree. For a specific diagnostic sequence, two signals detected by a receiver antenna were recorded; in case 1: the conductive probe is inserted into a hole of the MHA, and in case 2: the conductive probe is removed from the MHA. By repeating 
the process, the signals in case 1 and case 2 of all the used holes shown in Figure 13 were obtained. Subtracting the signal of case 2 from that of case 1 for each hole, the differential signals of all the holes create one signal data set with a spatial profile. The processes described above were conducted for the following two series; for data set A; a sample (cylindrical aluminum stick, radius $1.6 \mathrm{~mm}$, height $110 \mathrm{~mm}$ ) on the MHA and for data set B; the sample was removed from the MHA. Finally, the one-dimensional imaging map was completed by subtracting the signals of data set A from those of data set B. The sample, and the results as an example are shown in Figure 13.

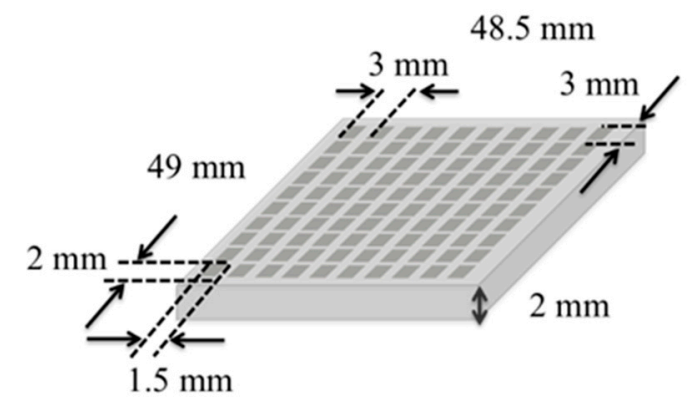

Figure 11. MHA of the imaging experiments.

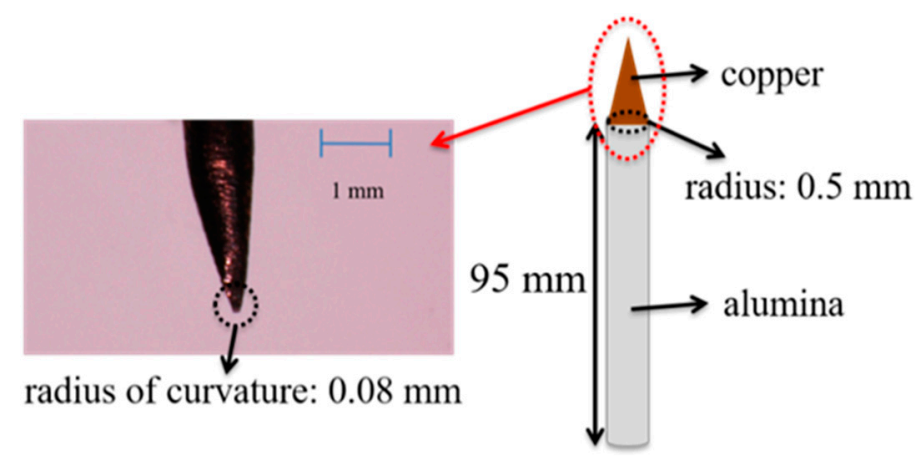

Figure 12. The conductive probe for localized electromagnetic distortion.

(a)

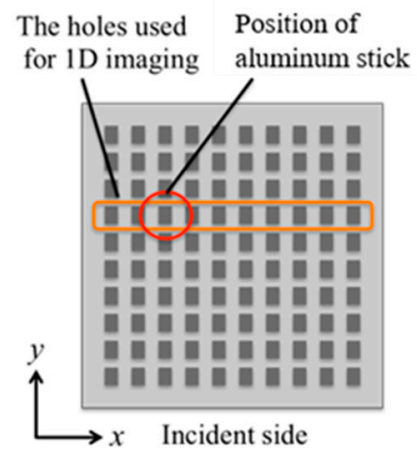

(b)

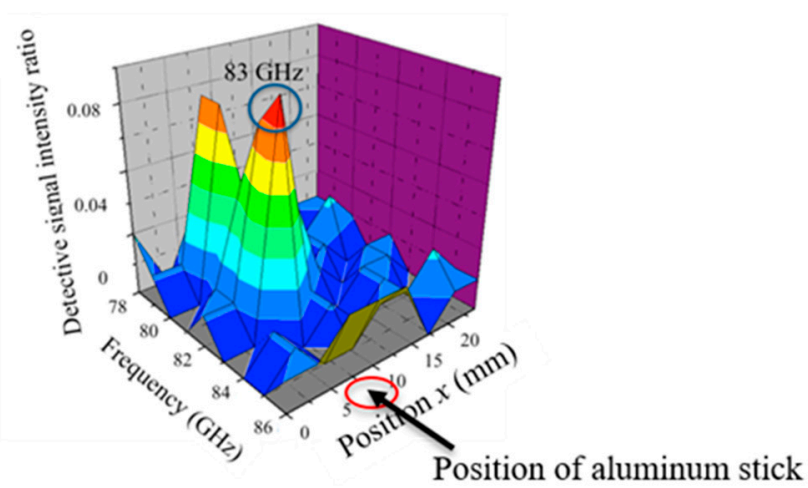

Figure 13. (a) The holes used for one-dimensional imaging; (b) the result of one-dimensional imaging for incident waves of 78-86 GHz.

Figure 13 shows that the detected signals of a conductive sample obtained at $x \sim 8 \mathrm{~mm}$, which corresponds to the location of the sample, are remarkable when electromagnetic waves in the frequency range of 78-80 GHz and around $83 \mathrm{GHz}$ are incident. It also indicated that other waves cannot detect significant signals. Since the incident angle at each hole of the MHA differs slightly from the reflective angle in the system, detection capacity might depend on the frequency of incident waves to some 
degree. For this reason, using a longer distance from the MHA to the transmitter and receiver antennas may provide greater uniformity in SSPPs generated on the MHA. From the results shown in Figure 13, we fixed the operation frequency at $83 \mathrm{GHz}$.

After the selection of the wave frequency based on the one-dimensional experiment, we performed experiments for two-dimensional permittivity imaging. Three samples with different permittivity values were provided, and we used them for two-dimensional imaging experiments, using an experimental system similar to that shown in Figures 10-12. Similar to the one-dimensional imaging experiments, the samples were set up on the MHA, and optimal signals were assured by executing the process described above (two procedures for data sets A and B in which cases 1 and 2 are for profiling). The samples included one conductive material, copper sticks, and a dielectric solid and liquid, an alumina stick and DMSO (dimethyl sulfoxide). The values of relative permittivity of alumina and DMSO are 9.9 and 43 - j10.9, respectively, in the millimeter-wave band. DMSO works as a biomaterial phantom in our experiment, since its permittivity is nearly equal to that of the human body [32]. To contain the liquid DMSO in the setup, an acrylic capsule array was used as a supporting experimental tool. Therefore, when comparing signals, in data set A the sample was on and in; in data set $\mathrm{B}$, the sample was removed from the MHA and an acrylic capsule array was set up in the same position on the MHA in both cases. The results of the two-dimensional imaging experiments for these three types of sample are shown in Figures 14-17.

Arrangement of the samples
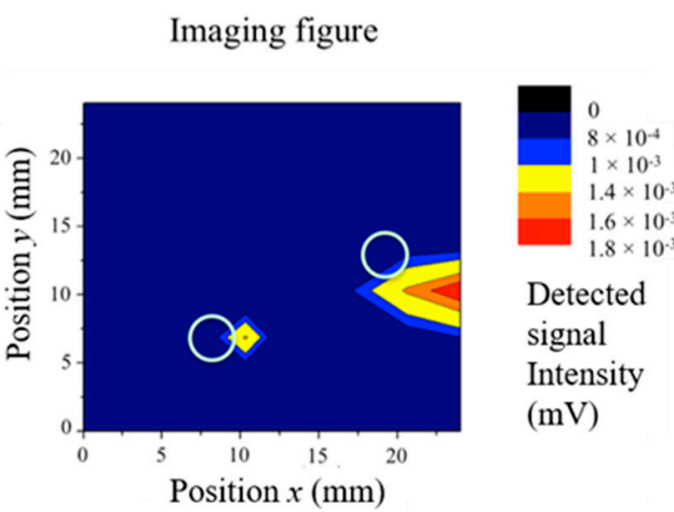

Detected signal Intensity

Position $x(\mathrm{~mm})$ $(\mathrm{mV})$

\section{Sample: two copper sticks $(2 \mathrm{~mm} \times 2 \mathrm{~mm} \times 30 \mathrm{~mm})$}

Figure 14. Two-dimensional imaging of conductive samples (two copper sticks).

Arrangement of the samples

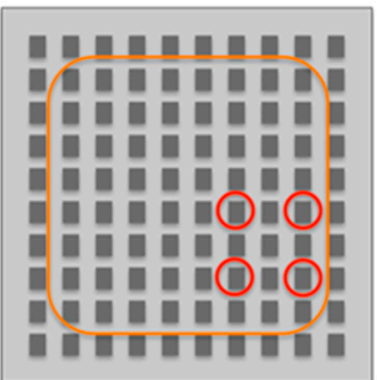

Imaging figure

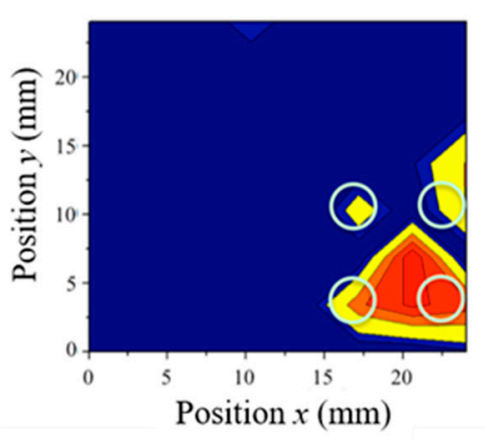

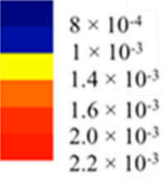

Detected signal Intensity $(\mathrm{mV})$

Sample: four copper sticks $(2 \mathrm{~mm} \times \mathbf{2} \mathbf{~ m m} \times \mathbf{3 0} \mathbf{~ m m})$

Figure 15. Two-dimensional imaging of conductive samples (four copper sticks). 
Arrangement of the samples

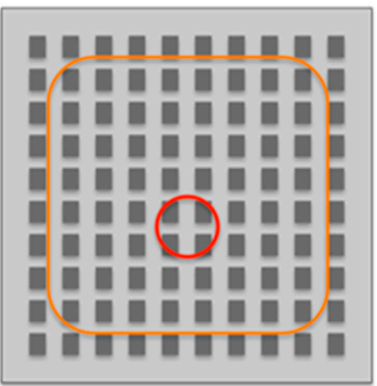

Imaging figure

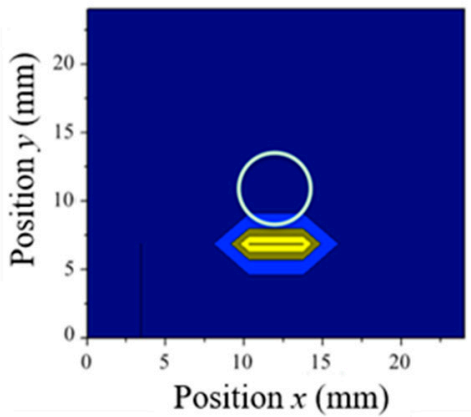

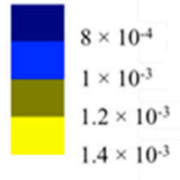

Detected

signal

Intensity

$(\mathrm{mV})$

\section{Sample: an alumina stick (radius: $3 \mathrm{~mm}$, height: $61 \mathrm{~mm}$ )}

Figure 16. The two-dimensional imaging of dielectric samples (an alumina stick).

Arrangement of the samples

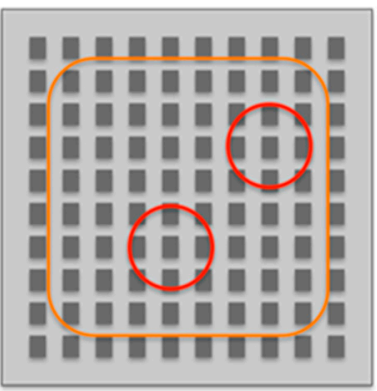

Imaging figure

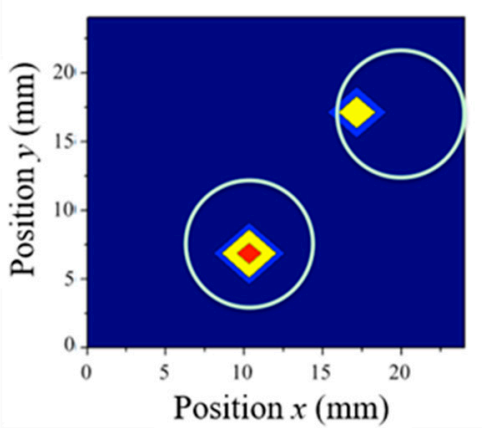

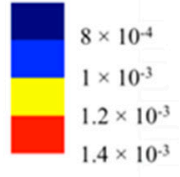

Detected signal Intensity $(\mathrm{mV})$

\section{Sample: DMSO (liquid, $150 \mu \mathrm{L} \times 2$, in an acrylic case)}

Figure 17. Two-dimensional imaging of dielectric samples (DMSO).

In Figures 14 and 15, although the positions of the detected signals are slightly different from the actual positions of the samples, the existence of the conductive samples was clearly detectable and related to their position. Also, in Figures 16 and 17, the results confirm the detection of dielectric samples at almost their proper positions even when the positions of the samples varied, though DMSO is a lossy material. These results show the effectiveness of the use of concentrated electric distributions for permittivity signal detection in this scheme, which differs from conventional methods of signal detection in the microwave band. Also, comparing the results of the conductive samples (Figures 14 and 15) to those of the dielectric samples (Figures 16 and 17), the positions of the detected signals of the former are less accurate than those of the latter. Considering the fact that the electric distributions of SSPPs are distorted to a greater extent by conductors than by dielectrics, the detection accuracy in the latter case may be superior to that in the former case. Furthermore, Figures 14 and 15 suggest that the detection accuracy for samples near corners is low in comparison with that of samples on inner locations. Since, in these experiments, the MHA has a limited size and SSPPs on the MHA are reflected at its corners, the electric distribution of SSPPs near corners tends to be non-uniform. Therefore, the distributions in the vicinity of its corners are not stable, and the detection accuracy of samples on corners tends to be low. By using an MHA with a larger number of holes, the detection capacity might be sufficiently accurate for practical use. On the other hand, the maximum values of the detected signal intensities of conductive samples are more intense than those of dielectric samples. In general, electric fields are affected more by conductors than dielectrics because of the boundary condition on the surface of conductors. Since DMSO has the large value of the imaginary part, it is consistent with the fact that the average value of the detected signal intensities in the case of the alumina sample was larger than that of DMSO. However, sufficient signal intensities were monitored in both cases for 
practical imaging, since the intensities satisfied the dynamic ranges of dB-order in the experiments. The results in all the cases confirm that the proper signals are obtainable at a mm-order distance by using the proposed method. This is an advantage over the conventional sensing method in which lasers or other strong light sources are required in the optical range.

The above discussions support the feasibility of a biomedical diagnosis that uses the proposed system. Finally, the result of the biomedical diagnosis in the case of a rat lung is reported. As shown in Figure 18, the results confirm a detection of the sample in more than half of the area. Although there is some slight signal misdetection, it shows that two-dimensional dialectic responses of the biomedical sample can be obtained in the proposed system.

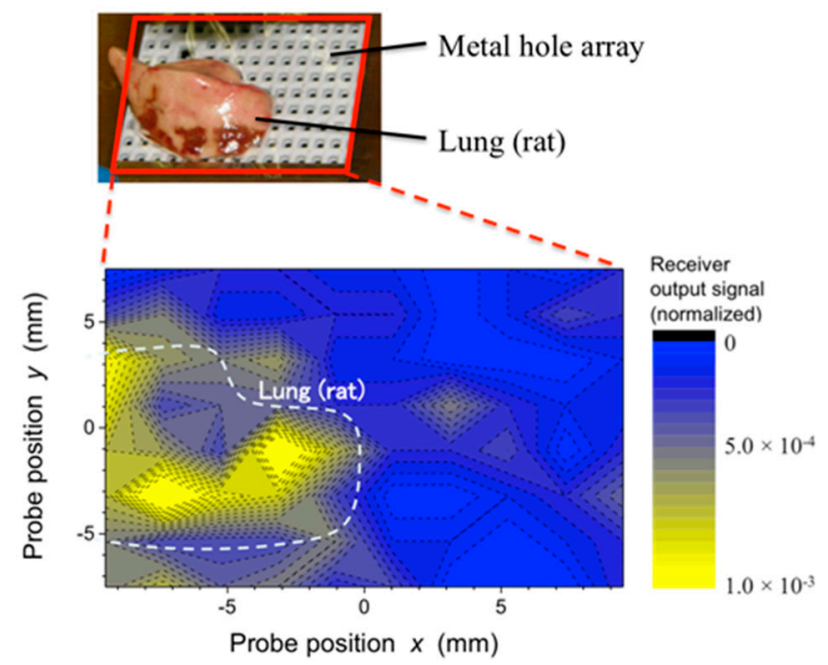

Figure 18. The result of biomedical diagnosis by using an MHA with rectangular holes (the size is $1.8 \mathrm{~mm} \times 1.4 \mathrm{~mm}$, the periods of each direction are $2.5 \mathrm{~mm}, 2 \mathrm{~mm}$ and the thickness is $2.3 \mathrm{~mm}$ ) in the case of a rat lung.

The above discussions and results indicate that the proposed method can be used for 2D imaging of permittivity distribution, and may be useful for biomedical diagnosis after optimization as a medical facility tool.

\section{Conclusions}

A 2D imaging method is proposed in this study, based on a meta-structure with a scanning defect, using a metal hole array (MHA) and a conductive probe (a needle-like conductor) in the millimeter-wave range. In order to validate the proposed method, a theoretical model of SSPP generation on an MHA was introduced, numerical electromagnetic analyses of localized distortions of the electric fields on and around the MHA were conducted, and one- and two-dimensional imaging experiments using conductive and dialectic samples verified the theoretical predictions.

In Section 2, the dispersion relation of SSPPs in the case where the MHA has rectangular holes with oblique incidence of sampling waves was derived, which indicated the physical phenomenon of SSPP generation on an MHA. In Section 3, with the use of HFSS, the transmission and reflection properties of an MHA were analyzed when millimeter waves were injected into the structure, and this analysis implied that SSPP modes exist under the cut-off frequency in our MHA model. In addition, the electromagnetic distributions around an MHA with an inserted conductive probe were compared with those of an MHA without the probe, confirming the difference in the electric distributions around the MHA in the two cases. Specifically, insertion of a conductive probe into an MHA can be useful for localized electromagnetic distortion. In Section 4, using an MHA and a conductive probe, a one-dimensional experiment was performed and confirmed the frequency dependency of the detected signal intensity in the range of $78-86 \mathrm{GHz}$. This showed that a wave at $83 \mathrm{GHz}$ can be 
monitored, producing the most intense level of detected signals. Then, two-dimensional imaging experiments with conductive samples (copper sticks) and dielectric samples (an alumina stick and DMSO (liquid)) were performed with the use of optimal waves at $83 \mathrm{GHz}$. Consequently, it was confirmed that the proposed measurement system allows the detection of the positions of conductive and dielectric samples by comparing the intensity levels of reflected signals with and without the samples. Finally, we demonstrated a biomedical diagnosis in the case of a rat lung by using the system. The result shows that although there is slight signal misdetection, two-dimensional dialectic responses of the biomedical sample can be obtained using the proposed method. Therefore, the proposed method has the potential for use in the two-dimensional imaging of permittivity distribution, such as in the biomedical task of localized tumor detection.

Author Contributions: G.I. performed the experiments and theoretical analyses shown here and wrote the paper by drawing the figures. O.S. conceived of and designed the research activities, as well as wrote the paper. Y.H. accelerated the study by presenting the technical issues in the medical point of view.

Funding: This research was partly funded by Project for Kyoto Bio-industry Creation \& Support, and by JSPS Kakenhi with grant number $18 \mathrm{H} 03690$.

Acknowledgments: The author thanks Y. Nishio and A. Iwai at Kyoto University for their fruitful comments on this study.

Conflicts of Interest: The authors declare no conflict of interest.

\section{References}

1. Hasch, J.; Topak, E.; Schnabel, R.; Zwick, T.; Weigel, R.; Waldschmit, C. Millimeter-wave Technology for Automotive Radar sensors in the 77 GHz Frequency Band. IEEE Trans. Microw. Theory Tech. 2012, 60, 845-860. [CrossRef]

2. Theofanopoulos, P.C.; Trichopoulos, G.C. Modeling of $\mathrm{mm} \mathrm{W}$ and THz Imaging Systems Using Conjugate Field Coupling. IEEE Antennas Wirel. Propag. Lett. 2018, 17, 213-216. [CrossRef]

3. Ghasr, M.T.; Horst, M.J.; Dvorsky, M.R.; Zoughi, R. Wideband Microwave Camera for real-Time 3-D Imaging. IEEE Trans. Antennas Propag. 2017, 65, 258-268. [CrossRef]

4. Long, F.; He, M.; Shi, H.C.; Zhu, A.N. Development of evanescent wave all-fiber immunosensor for environmental water analysis. Biosens. Bioelectron. 2008, 23, 952-958. [CrossRef] [PubMed]

5. Katyal, J.; Soni, R.K. Localized Surface Plasmon Resonance and Refractive Index Sensitivity of Metal-Dielectric-Metal Multilayered Nanostructure. Plasmonics 2014, 9, 1171-1181. [CrossRef]

6. Tiwari, K.; Sharma, S. Surface plasmon based sensor with order-of-magnitude higher sensitivity to electric field induced changes in dielectric environment at metal/nematic liquid-crystal interface. Sens. Actuators A 2014, 216, 128-135. [CrossRef]

7. Zhu, Q.; Tan, W.; Wang, Z. The combined effect of side-coupled gain cavity and lossy cavity on the plasmonic response of metal-dielectric-metal surface plasmon polariton waveguide. J. Phys. Condens. Matter 2014, 26, 255301. [CrossRef] [PubMed]

8. Strachko, A.A.; Agranovich, V.M. To the theory of surface plasmon on metals covered with resonant thin films. Opt. Commun. 2014, 332, 201-205. [CrossRef]

9. Anker, J.N.; Hall, W.P.; Lyandres, O.; Shah, N.C.; Zhao, J.; Duyne, R.P.V. Biosensing with plasmonic nanosensors. Nat. Mater. 2008, 7, 442-453. [CrossRef]

10. Kreno, L.E.; Leong, K.; Farha, O.K.; Allendorf, M.; Duyne, R.P.V.; Hupp, J.T. Metal-Organic Framework Material as Chemical Sensors. Chem. Rev. 2012, 112, 1105-1125. [CrossRef]

11. Camden, J.P.; Dieringer, J.A.; Zhao, J.; Duyne, R.P.V. Controlled Plasmonic Nanostructures for Surface-Enhanced Spectroscopy and Sensing. Acc. Chem. Res. 2008, 41, 1653-1661. [CrossRef] [PubMed]

12. Pendry, J.B.; Moreno, L.M.; Vidal, F.J.G. Mimicking Surface Plasmon with Structured Surfaces. Science 2004, 305, 847-848. [CrossRef] [PubMed]

13. Woolf, D.; Kats, M.A.; Capasso, F. Spoof surface plasmon waveguide forces. Opt. Lett. 2014, 39, 517-520. [CrossRef] [PubMed]

14. Bhattacharya, S.; Shah, K. Multimodal propagation of the electromagnetic wave on a structured perfect electric conductor (PEC) surface. Opt. Commun. 2014, 328, 102-108. [CrossRef] 
15. Shen, L. Effect of absorption on terahertz surface plasmon polaritons propagating along periodically corrugated metal wires. Phys. Rev. B 2008, 77, 075408. [CrossRef]

16. Ng, B.; Hanham, S.M.; Wu, J.; Dominguez, A.I.F.; Klein, N.; Liew, Y.F.; Breese, M.B.; Hong, M.; Maier, S.A. Broadband Terahertz Sensing on Spoof Plasmon Surfaces. ACS Photonics 2014, 1, 1059-1067. [CrossRef]

17. Miyamaru, F.; Hangyo, M. Anomalous terahertz through double-layer metal hole arrays by coupling of surface plasmon polaritons. Phys. Rev. B 2005, 71, 165408. [CrossRef]

18. Miyamaru, F.; Hayashi, S.; Otani, C.; Kawase, K. Terahertz surface-wave resonant sensor with a metal hole array. Opt. Lett. 2006, 31, 1118-1120. [CrossRef] [PubMed]

19. Miyamaru, F.; Kamijyo, M.; Hanaoka, N.; Takeda, M.W. Controlling extraordinary transmission characteristics of metal hole arrays with spoof surface plasmons. Appl. Phys. Lett. 2012, 100, 081112. [CrossRef]

20. Miyamaru, F.; Hangyo, M. Finite size effect of transmission property for metal hole arrays in subterahertz region. Appl. Phys. Lett. 2004, 84, 2742-2744. [CrossRef]

21. Miyamaru, F. Effect of hole diameter on terahertz surface-wave excitation in metal hole arrays. Phys. Rev. B 2006, 74, 153416. [CrossRef]

22. Brissova, M.; Fowler, M.J.; Nicholson, W.E.; Chu, A.; Hirshberg, B.; Harlan, D.M.; Powers, A.C. Assessment of Human Pancreatic Islet Architecture and Comparison by Laser Scanning Confocal Microscopy. J. Histochem. Cytochem. 2005, 53, 1087-1097. [CrossRef] [PubMed]

23. Klein, A.E.; Janunts, N.; Steinert, M.; Tunnermann, A.; Pertsch, T. Polarization-Resolved Near-Field Mapping of Plasmonic Aperture Emission by a Dual-SNOM System. Nano Lett. 2014, 14, 5010-5015. [CrossRef] [PubMed]

24. Itami, G.; Sakai, O. Symmetrical Estimation Method for Skin Depth-Control of Spoof Surface Plasmon by Using Dispersed Waves from Metallic Hole Array. J. Appl. Phys. under review.

25. Meltzer, C.C.; Prlce, J.C.; Mathis, C.A.; Greer, P.J.; Cantwell, M.N.; Houck, P.R.; Mulsant, B.H.; Ellezer, D.B.; Loprestl, B.; Dekosky, S.T.; et al. PET Imaging of Serotonin Type 2A Receptors in Late-Life Neuropsychiatric Disorders. Am. J. Psychiatry 1999, 156, 1871-1878. [PubMed]

26. Haim, S.B.; Murthy, V.L.; Breault, C.; Allie, R.; Sitek, A.; Roth, N.; Fantony, J.; Moore, S.C.; Park, M.A.; Kijewski, M.; et al. Quantification of Myocardial Perfusion Reserve Using Dynamic SPECT Imaging in Humans: A Feasibility Study. J. Nucl. Med. 2013, 54, 873-879. [CrossRef] [PubMed]

27. Juette, M.F.; Gould, T.J.; Lessard, M.D.; Mlodzianoski, M.J.; Nagpure, B.S.; Bennett, B.T.; Hess, S.T.; Bewersdorf, J. Three-dimensional sub-100 nm resolution fluorescence microscopy of thick samples. Nat. Methods 2008, 5, 527-529. [CrossRef] [PubMed]

28. Kubota, S.; Xiao, X.; Sasaki, N.; Kayaba, Y.; Kimoto, K.; Moriyama, W.; Kozaki, T.; Hanada, M.; Kikkawa, T. Confocal Imaging Using Ultra Wideband Antenna Array on Si Substrates for Breast Cancer Detection. Jpn. J. Appl. Phys. 2010, 49, 097001. [CrossRef]

29. Vidal, F.J.G.; Moreno, L.M.; Pendry, J.B. Surfaces with holes in them: New plasmonic metamaterials. J. Opt. A Pure Appl. Opt. 2005, 7, S97-S101. [CrossRef]

30. Sekimoto, K.; Takayama, M. Negative ion formation and evolution in atmospheric pressure corona discharges between point-to-plane electrodes with arbitrary needle angle. Eur. Phys. J. D 2010, 60, 589-599. [CrossRef]

31. Itami, G.; Akiyama, T.; Sakai, O.; Harada, Y. Localized electromagnetic distortion in 2D metal hole array and its application to imaging of permittivity distribution. In Proceedings of the Asia-Pacific Microwave Conference (APMC), Sendai, Japan, 4-7 November 2014.

32. Urata, M.; Kimura, S.; Wakino, K.; Kitazawa, T. Optimization of three layer tapered coaxial line applicator for cancer thermotherapy by impedance matching of transition layer sections. In Proceedings of the IEEE International Symposium on Antennas and Propagation (APSURSI), Spokane, WA, USA, 3-8 July 2011.

(C) 2019 by the authors. Licensee MDPI, Basel, Switzerland. This article is an open access article distributed under the terms and conditions of the Creative Commons Attribution (CC BY) license (http:/ / creativecommons.org/licenses/by/4.0/). 